\section{$\underset{\substack{\text { hommes } \\ \text { \& migrations }}}{ }$}

\section{Hommes \& migrations}

Revue française de référence sur les dynamiques

migratoires

$1288 \mid 2010$

Langues et migrations

\title{
Petits-enfants de migrants italiens et espagnols en Suisse
}

Relations familiales et pratiques langagières

\section{Rosita Fibbi et Marinette Matthey}

\section{(2) OpenEdition \\ Journals}

Édition électronique

URL : http://journals.openedition.org/hommesmigrations/857

DOI : 10.4000/hommesmigrations.857

ISSN : 2262-3353

Éditeur

Musée national de l'histoire de l'immigration

Édition imprimée

Date de publication : 1 novembre 2010

Pagination : 58-69

ISSN : $1142-852 X$

\section{Référence électronique}

Rosita Fibbi et Marinette Matthey, "Petits-enfants de migrants italiens et espagnols en Suisse », Hommes \& migrations [En ligne], 1288 | 2010, mis en ligne le 29 mai 2013, consulté le 30 avril 2019. URL : http://journals.openedition.org/hommesmigrations/857 ; DOI : 10.4000/

hommesmigrations.857 


\section{Petits-enfants de migrants italiens et espagnols en Suisse Relations familiales
et pratiques langagières}

Par Rosita Fibbi, Forum suisse pour l'étude des migrations et de la population, université de Neuchâtel et Marinette Matthey, LIDILEM, université de Grenoble

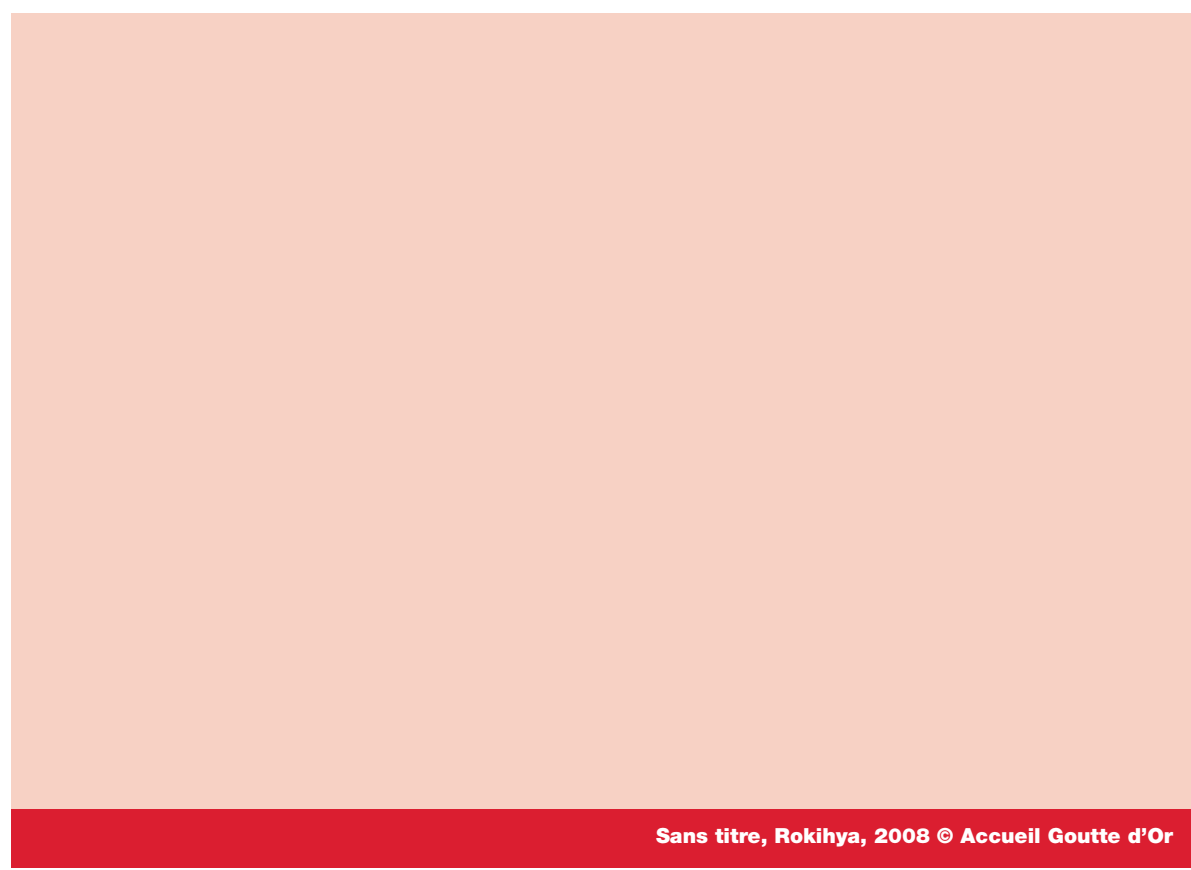

En Suisse, où sont reconnues quatre langues nationales, les migrants d'origine italienne et espagnole font de leur héritage linguistique une richesse. La famille constitue le cadre privilégié de la transmission de l'identité et de la langue d'origine des migrants. La génération des grands-parents est souvent investie de la mission de perpétuer le bilinguisme familial chez les petits-enfants. Si le partage de cette langue des origines chargée d'émotions sert à affermir les liens familiaux, il offre également aux jeunes une compétence valorisée au sein d'une société plurilingue. 
L'intégration des migrants dans leur nouvelle société de résidence est un processus de longue haleine qui se déroule non pas au cours d'une vie mais plutôt dans la succession des générations. Plusieurs indicateurs sont généralement retenus dans la littérature pour rendre compte du processus de changement individuel et collectif concernant les migrants et leurs familles : niveau de formation, insertion sur le marché du travail, intermariage, comportement démographique, pour ne citer que les plus usités. Nous nous intéressons ici aux dynamiques relatives aux langues en présence, celle(s) dites d'origine et la ou les langue(s) locale(s), étant entendu qu'elle(s) assume(nt) à la fois des fonctions communicatives et identitaires (identité personnelle et collective). L'approche des questions linguistiques sur trois générations est cohérente avec la temporalité longue des processus d'incorporation des populations immigrées, comme le montre l'expérience américaine en la matière.

La littérature américaine a résumé le changement des pratiques linguistiques sur le long terme dans le modèle d'anglicisation sur trois générations : selon Joshua Fishman $^{(1)}$, la perte de la langue d'origine intervient sur trois générations, de sorte que le bilinguisme est un pont intergénérationnel temporaire relativement peu important entre le monolinguisme en langue d'origine (LO) des immigrants eux-mêmes et le nouveau monolinguisme dans la langue locale du pays d'immigration (LL) de leurs petits-enfants. Ce modèle ne tient pas compte de l'observation déjà faite par Markus Hansen $^{(2)}$, selon lequel les jeunes de la troisième génération $(\mathrm{G} 3)$ désirent - à tout le moins - s'approprier ce que leurs parents (G2) ont volontairement mis entre parenthèses, notamment la langue d'origine de leurs grands-parents (G1), dans leur recherche d'une plus grande acceptation de la part de la société d'immigration.

Il est légitime de se demander dans quelle mesure ces observations du contexte américain peuvent être aussi valables de ce côté-ci de l'Atlantique, dans une Europe en construction, où la pluralité linguistique constitue un trait spécifique de cet ensemble économique et social, le plurilinguisme est aujourd'hui largement reconnu et valorisé. C'est que, dans les dernières décennies, la perception du bilinguisme s'est profondément modifiée : de décrié qu'il était jusqu'au début des années soixante, le bilinguisme est désormais crédité de stimuler le développement cognitif individuel et le plurilinguisme devient le support de la cohésion sociale dans les entités politiques composites que sont devenues nos sociétés.

Finalement, la théorie du retour à un monolinguisme au bout de trois générations de migrants suggère qu'il s'agit d'un processus "naturel" de l'écoulement du temps, plutôt que le fruit de politiques spécifiques visant à poursuivre avec les migrants d'aujourd'hui ce processus d'homogénéisation linguistique de l'espace national commencé jadis avec la construction des États-nations. Dans un monde de plus en plus interconnecté sur les plans de la communication et de la mobilité des personnes, 
le monolinguisme ne semble plus l'horizon nécessaire des populations, qu'elles soient d'ailleurs d'origine immigrée ou non.

Il n'est guère contesté qu'à long terme les immigrés et leurs descendants se tournent vers la pratique dominante de la LL. Il s'agit plutôt de cerner si et dans quelles conditions celle-ci est accompagnée du maintien de la langue d'origine que ce soit dans le contexte familial ou extra-familial. Le contexte suisse se prête tout particulièrement à l'étude de ces dynamiques langagières étant donné sa longue histoire de plurilinguisme à l'échelle nationale et la présence sur son sol de migrants depuis de nombreuses décennies.

\section{Questions de recherche}

À la lumière de ces considérations, nous avons étudié ${ }^{(3)}$ la transmission de la langue d'origine des migrants, à la fois comme répertoire de communication et comme marqueur identitaire. Notre intérêt s'est porté sur les Italiens et Espagnols et leurs descendants car ce sont les deux seuls groupes d'immigrés stabilisés de longue date qui ont une troisième génération aujourd'hui en âge d'adolescence en Suisse. Ainsi avonsnous étudié les stratégies de transmission linguistique développées par les enfants de migrants (deuxième génération, G2) et leurs parents (primo-migrants, G1) en direction de la troisième génération (G3) ainsi que les pratiques langagières de ces jeunes. La présence des grands-parents, leur implication dans la prise en charge des petits-enfants ainsi que la fréquence non négligeable des modes de vie transfrontaliers de cette génération donnent aux compétences plurilingues une résonance affective et pratique susceptible d'influencer les objectifs des transmissions linguistiques et culturelles ainsi que les dispositions des jeunes en la matière.

Partant de l'hypothèse que ces groupes immigrés de longue date sont aujourd'hui bien acceptés par la population suisse, que le statut des langues italienne et espagnole en Suisse et dans le monde est relativement élevé, et que le bi/plurilinguisme est aujourd'hui valorisé et associé aux modes de vie transnationaux, nous postulons que parmi les jeunes de la troisième génération en Suisse ("les G3"), nous trouvons effectivement des pratiques bi ou même plurilingues et des identités multiples. Ces pratiques relèvent d'un bilinguisme fonctionnel, basé sur des compétences partielles, permettant aux individus d'atteindre des objectifs communicationnels en contexte dans deux ou plusieurs langues.

Une des difficultés théoriques et pratiques dans notre étude a été de désigner les langues en présence. Dans les premiers travaux sur les aspects langagiers de la migration, ces langues sont désignées habituellement comme "langue d'origine" et 
"langue d'accueil ${ }^{(4) "}$. Cette dernière dénomination paraît inadéquate en référence à des personnes nées et ayant grandi en migration, comme c'est le cas pour les G2 et G3 ; nous parlerons dès lors de "langue locale". Pour la dénomination de la langue des grands parents, nous conservons le terme "langue d'origine" utilisé dans les travaux mentionnés ci-dessus, tout en sachant que cette expression recouvre un continuum de variétés allant d'un pôle dialectal à un pôle standard. La dénomination "langue d'origine" peut encore se justifier pour les parents (G2) qui ont souvent appris et beaucoup pratiqué cette langue tout au moins dans leur jeunesse. Elle est en revanche moins évidente pour les G3 car elle désigne la langue de leurs grands-parents ; la notion de "langue héritée" paraît plus pertinente à leur égard. Repris de l'anglais heritage language $(\mathrm{HL})^{(5)}$, ce terme désigne une (variété de) langue apprise dans l'entourage familial, généralement dans la socialisation primaire, qui est différente de la (variété de) langue locale. Une langue héritée est la langue associée au background culturel de la personne ${ }^{(6)}$. Par souci de cohérence à l'intérieur d'un texte traitant de l'évolution intergénérationnelle de l'usage des langues, la langue des grands-parents est désignée comme LOH, "langue d'origine et héritée".

Dans le but de tester nos hypothèses, notre projet s'est intéressé aux pratiques de transmission de la langue d'origine chez les G1 et G2, à la présence de la LOH dans le répertoire des G3 et à leurs pratiques langagières ainsi qu'à la signification identitaire que les pratiques plurilingues pourraient avoir pour ces jeunes.

La recherche s'est basée sur des entretiens semi-directifs dans 32 familles habitant les agglomérations de Genève et Bâle. Dans chaque famille nous avons interrogé un représentant de chaque génération : les grands-parents (G1), les parents (G2) et les adolescents (G3). Les entretiens étaient menés par des enquêtrices bilingues, laissant ainsi à la personne interrogée le loisir de passer de la langue d'origine/langue héritée à la langue locale; ils portaient sur la biographie langagière, les pratiques linguistiques ainsi que sur les attitudes envers différentes langues et envers le plurilinguisme.

\section{Les relations et la transmission intergénérationnelles}

Nous observons des relations familiales particulièrement denses dans les familles (G1 et G2) d'origine italienne et espagnole ${ }^{(7)}$. Elles se concrétisent, par exemple, dans une fréquente proximité d'habitation, un rythme élevé et régulier de rencontres, une entraide importante avec échange soutenu de services réciproques entre générations, un flux de richesse des parents vers les enfants ainsi qu'une forte mobilisation familiale en vue de l'affirmation professionnelle de la génération cadette. Autant de 
caractéristiques spécifiques aux familles d'origine immigrée qui ne trouvent pas d'équivalent dans les familles suisses ${ }^{(8)}$.

Ce type de relations intergénérationnelles n'est pas un trait indélébile dont les immigrés seraient porteurs en raison de leurs origines culturelles. Car les formes familiales varient notamment en raison de facteurs contextuels, comme l'indique Cigdem Kagitcibasi, avec son modèle du changement familial (MFC) ${ }^{(9)}$. Elle en identifie trois qui sont directement influencés par le contexte économique du pays où vit la famille. Dans chacun de ces modèles on observe un style parental et des pratiques de

\section{Les grands-parents en migration sont considérés comme des "dépositaires de cultures", soucieux d’assurer une forme élargie de reproduction culturelle, notamment en ce qui concerne la transmission de la langue d’origine.} socialisation spécifiques. Les familles dans le passage d'un contexte rural à un contexte urbain, et notamment en migration, développent un modèle qu'elle qualifie d'“interdépendance émotionnelle", caractérisé par un affaiblissement marqué de l'interdépendance de type économique mais une permanence des liens émotionnels forts. Les pratiques éducationnelles visent aussi bien un contrôle des enfants pour que ceux-ci gardent un contact étroit avec la famille que des formes de promotion de l'autonomie des enfants. L'autonomie n'est plus une menace pour la famille, au contraire, elle est promue et soutenue parce que plus fonctionnelle que l'obéissance à l'adaptation des styles de vie liés à l'urbanisation. Les personnes socialisées dans ces familles maintiennent un sens aigu de l'appartenance et en même temps ont une orientation claire vers l'autonomie.

C'est dans ce rapport à la fois fidèle et malléable à la tradition que l'on comprend les choix de la langue à transmettre : les primo-migrants (G1), en effet, ont transmis à leurs enfants (G2) non pas le dialecte régional, qui constitue dans bien des cas leur véritable langue, mais la langue standard, apprise bien souvent une fois à l'étranger, "hors sol" en quelque sorte, en côtoyant leurs compatriotes originaires d'autres régions. L'abandon du dialecte est pour eux une renonciation lucide qui prend son sens en vue de la mobilité sociale escomptée à tout le moins pour leurs enfants ${ }^{(10)}$. La relation des primo-migrants à leurs petits-enfants se situe dans le même souci de conjuguer tradition et exigences présentes.

Les grands-parents des familles que nous avons rencontrées sont assez présents dans la vie de leurs petits-enfants, surtout lorsqu'ils sont en bas âge mais également à l'adolescence, dans un rapport qui prolonge les relations d'échanges pratiques et symboliques avec leurs propres enfants adultes. Les grands-parents en migration sont considérés comme des “dépositaires de cultures", soucieux d'assurer une forme élargie 
de reproduction culturelle, notamment en ce qui concerne la transmission de la langue d'origine. Si la transmission de la $\mathrm{LOH}$ va souvent de soi dans la relation entre G1 et G2, elle fait en revanche toujours l'objet d'une élaboration explicite dans la relation entre les grands-parents et les petits-enfants. Le choix de la langue standard est encore plus clairement réaffirmé en cas de transmission linguistique aux G3 : les grandsparents s'orientent vers la langue standard pour transmettre leur langue d'origine.

La mobilisation des grands-parents est d'autant plus cruciale pour la transmission linguistique lorsque les petits-enfants ont des parents ne parlant pas la même langue d'origine et où, en conséquence, la pratique de la LOH ne va pas de soi. Si toutefois elle s'avère, c'est bien souvent dû à la volonté des grands-parents, qui mettent tout en ceuvre pour apprendre et soutenir la motivation de leurs petits-enfants ${ }^{(11)}$. Cette posture d'initiative volontariste se rencontre plus souvent chez les grands-parents ayant un niveau élevé de formation mais aussi chez des cadres associatifs quel que soit leur niveau de formation. Parfois la mobilisation des grands-mères (ce sont elles qui assurent généralement ce rôle) correspond à un besoin personnel : "La vie m'a donné cet héritage... je cherche à le transmettre à mes petits-enfants", affirme cette grand-mère qui dit s'efforcer d'inventer des occasions multiples d'apprentissage voire d'immersion linguistique pour ses petits-enfants qui grandissent dans une famille où les parents ne partagent pas la même langue d'origine.

\section{La langue en héritage}

Le rôle des grands-parents dans la transmission linguistique découle le plus souvent d'une investiture, d'une délégation de la part de leurs enfants ${ }^{(12)}$ qui souhaitent perpétuer le bilinguisme familial mais ne se sentent pas en mesure d'assurer l'apprentissage de la langue d'origine aux petits. Si virtuellement tous les enfants de migrants interrogés (G2) ont reçu la LOH de leurs parents et parlent la langue de leurs parents, ils ne se considèrent pas tous comme des locuteurs suffisamment habiles pour la parler au quotidien à l'âge adulte. La langue locale remplit toutes les fonctions pragmatiques nécessaires pour vivre dans la communauté et le maintien de la $\mathrm{LOH}$ ne peut se faire qu'au prix d'un investissement particulier auquel les grands-parents sont priés d'apporter leur concours.

La majorité des grands-parents s'acquitte volontiers de cette tâche qui résonne positivement en eux, mais ils ne sont pas prêts à y sacrifier - voire à mettre en péril la spontanéité et la fraîcheur de la relation avec leurs petits-enfants ; face à un enfant qui serait réticent à parler la $\mathrm{LOH}$, les grands-parents adoptent la LL, devenue grâce aux petits-enfants aussi une langue avec laquelle ils peuvent exprimer de l'affection. 
C'est que la transmission est aussi réversible, car les aînés apprennent (et sont prêts à le faire) des cadets ! Il faut relever en outre que la langue du lieu s'est lentement mais sûrement introduite au fil du temps non seulement dans la vie professionnelle des G1 mais également dans l'intimité de ces adultes qui ont vécu si longtemps en migration. "Tout au début javais beaucoup de difficultés pour parler. Aujourd'hui encore jai un accent pas possible [...] mais moi je me sens à l'aise", dit cette grand-mère d'origine italienne.

La compétence linguistique en LOH n'est jamais recherchée comme une alternative à la compétence en LL; l'objectif manifeste des parents et grands-parents est le bilinguisme fonctionnel entre LL et LOH. Une fois assuré le premier arrimage symbolique aux racines familiales, la tâche de l'apprentissage pourra être demandée à d'autres instances, scolaires notamment. Dans ces cas alors, la transmission aura pour ambition de dépasser le cadre familial, de parvenir, par exemple, à une qualification sur le marché du travail ou alors à donner substance à une autre appartenance collective de l'individu, notamment la capacité de nouer des contacts extra-familiaux dans la région d'origine. Ce sera l'école qui familiarisera le jeune au "nous" culturel tel qu'élaboré dans le cadre national : ainsi certains grands-parents mentionnent l'apprentissage linguistique comme un outil pour apprivoiser l'histoire du pays, sa littérature ou sa richesse culturelle au-delà de sa seule fonction de communication.

\section{Les pratiques langagières des G3}

L'objectif d'une communication fluide est donc le but premier visé par les familles. La situation d'entretien permet d'entrevoir l'étendue des pratiques langagières et l'évolution intergénérationnelle de la langue d'origine/héritée. Dix-neuf de nos jeunes interlocuteurs (donc un peu plus de la moitié d'entre eux) savent manier la LOH dans une situation aussi peu naturelle qu'un entretien de recherche. La différence d'âge entre enquêtrice et jeune a pu évoquer des situations scolaires induisant un sentiment d'insécurité linguistique et incitant le jeune à produire des réponses limitées dans un souci de ne pas s'écarter de la norme scolaire ${ }^{(13)}$. Le passage à la langue locale paraît souvent associé à l'insécurité linguistique. Un large tiers de jeunes G3 disent ne guère avoir recours à la $\mathrm{LOH}$. Une majorité, en revanche, la pratique que ce soit dans le cadre de la famille restreinte ou élargie (voir tableau 1). Globalement, les pratiques langagières des jeunes de la troisième génération reflètent assez fidèlement les constellations familiales : maîtrise et pratique de la LOH sont en général moins assurées et moins fréquentes si l'un des parents n'est pas un locuteur natif de cette langue. Toutefois, le volontarisme peut parfois changer la donne et parmi les 18 enfants issus 


\section{Tableau 1. Usage de la LOH chez les jeunes G3}

\begin{tabular}{|l|c|c|c|c|c|}
\hline Usage de la LOH & $\begin{array}{c}\text { Italien } \\
\text { Bâle }\end{array}$ & $\begin{array}{c}\text { Italien } \\
\text { Genève }\end{array}$ & $\begin{array}{c}\text { Espagnol } \\
\text { Bâle }\end{array}$ & $\begin{array}{c}\text { Espagnol } \\
\text { Genève }\end{array}$ & TOTAL \\
\hline 1. dans la famille restreinte & 4 & 2 & 1 & 2 & 9 \\
2. dans la famille élargie & 4 & 2 & 1 & 3 & 10 \\
3. usage rare /inexistant & 2 & 6 & 3 & 2 & 13 \\
TOTAL & 10 & 10 & 5 & 7 & 32 \\
\end{tabular}

de couples mixtes du point de vue linguistique, certains (5) pratiquent la LOH même pendant l'entretien. Car d'autres facteurs influencent fortement les pratiques langagières des jeunes tels l'existence et l'intensité des liens avec la famille élargie ainsi que la richesse des expériences transnationales, qui font de cette $\mathrm{LOH}$ un véhicule de communication actuel et émotionnellement chargé.

L'ampleur du maintien de l'espagnol et de l'italien dans les familles descendantes de migrants ne peut être mesurée dans le cadre d'une étude qualitative ; elle apparaît néanmoins plus conséquente que ce que l'on a pu observer aux États-Unis, où la littérature scientifique sur le language mantainance and language shift souligne l'importance cruciale de la constellation familiale ${ }^{(14)}$. Nos résultats confortent notre hypothèse : bien que la pratique de la $\mathrm{LOH}$ diminue de manière évidente au cours des générations, on ne peut parler de monolinguisme dans la troisième génération d'origine italienne et espagnole en Suisse.

\section{La signification de la pratique de la LOH chez les petits-enfants de migrants}

Le processus de transmission n'aboutit que lorsque les receveurs acceptent de perpétuer les pratiques langagières (forcément modifiées) de leurs ascendants. Lacceptation de cet héritage se traduit par une compétence, de loin pas parfaite, de la langue des grands-parents, notamment dans nombre de familles fondées par les enfants de migrants. La transmission familiale est d'autant plus fréquente que les parents sont homoglottes mais elle n'est pas limitée à ce seul cas de figure.

Quelle signification attribuent les G3 aux pratiques plurilingues ? Les petits-enfants de migrants acceptent cet héritage linguistique des grands-parents et des parents car il fait sens dans le contexte d'immigration dans lequel ils vivent, qui valorise le 
plurilinguisme ${ }^{(15)}$. Cet aspect contextuel nous paraît capital : la construction de l'Europe, qui reconnaît sa grande diversité linguistique et la tradition suisse du plurilinguisme jouent un rôle important dans ces attitudes. Le discours très valorisant du plurilinguisme dans ce pays facilite l'actualisation de la compétence en langue d'origine/héritée dans la vie quotidienne des petits-enfants de migrants. Comme le dit une des adolescentes interrogées : "Les Suisses aussi ne parlent pas seulement l'allemand, ils parlent français, anglais. Mais il y a aussi les autres qui parlent allemand et turc, allemand et espagnol, allemand et portugais, jen connais beaucoup. Donc les jeunes de mon âge pour la plupart connaissent deux langues."

L'héritage linguistique accepté fait d'eux des sujets plurilingues dans un monde urbain qui se caractérise par le multilinguisme et par une valorisation de cette réalité, ou tout au moins une absence de stigmatisation

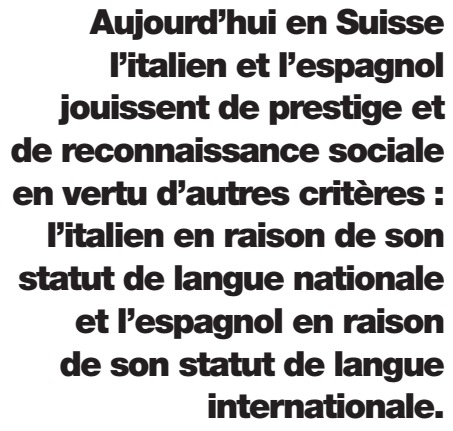
puisqu'il est répandu à la fois dans les minorités et dans la majoritée ${ }^{(16)}$.

La transmission familiale doit entrer en résonance avec le contexte de vie des jeunes pour pouvoir aboutir à une forme de reprise et de réinterprétation de l'héritage familial, à la hauteur des défis que les troisièmes générations sont appelées à relever dans leur vie quotidienne ${ }^{(17)}$. Cette résonance se nourrit notamment de facteurs institutionnels, tels que le prestige des langues en présence, la valorisation du bilinguisme et sa promotion, les opportunités d'apprentissage formalisé venant renforcer et stabiliser l'apprentissage informel familial.

Il ne faut pas perdre de vue que les langues dont il est question pour les jeunes interrogés dans cette étude ne sont pas que deux langues de la migration. Cela n'a pas toujours été le cas, toutefois aujourd'hui en Suisse l'italien et l'espagnol jouissent de prestige et de reconnaissance sociale en vertu d'autres critères : l'italien en raison de son statut de langue nationale et l'espagnol en raison de son statut de langue internationale. Comme telles, elles figurent en outre dans l'offre de formation des écoles secondaires supérieures.

Nos résultats valident ainsi la conception dynamique du travail de transmission et de son aboutissement proposée par Vatz Laaroussi : "La transmission vise à construire des identités nouvelles et adaptatives tout autant qu'à maintenir le fil d'une continuité symbolique et concrète dans des trajectoires de mouvements et de ruptures. Dans le contexte migratoire, les dynamiques de transmission sont à la jonction des identités individuelles et collectives tout autant que des espaces-temps du quotidien et de l'histoire ${ }^{(18)}$." 


\section{Conclusion}

En étudiant la transmission de la langue "héritée" il est apparu que les relations intergénérationnelles familiales observées dans les familles d'origine italienne et espagnole en Suisse se configurent selon le modèle d'interdépendance émotionnelle qui unit non seulement les parents et leurs enfants adultes mais également les petits-enfants jusqu'à l'adolescence. Cette configuration, favorisée par une régularité de contacts entre les trois générations propice à la transmission linguistique, promeut un environnement affectif favorable à la réception de cet héritage symbolique de la part des jeunes.

La spécificité d'une étude qualitative est de rendre intelligibles les configurations de comportements observées, car elle les restitue contextualisées dans un discours qui fait apparaître leurs motivations, leurs co-occurrences, leurs déterminations et leurs conséquences. Ces configurations sont généralisables au-delà du nombre restreint d'entretiens menés au cours de l'enquête alors que, bien évidemment, la mesure de la diffusion de ces configurations demeure hors de portée pour une pareille approche. On ne saurait donc généraliser ces résultats à l'ensemble des jeunes G3 en Suisse. Bien que nous nous soyons efforcées de diversifier l'échantillon de nos informateurs (notamment du point de vue de l'origine socioculturelle), nous sommes conscientes que l'acceptation même de la série d'entretiens a déjà introduit un certain biais favorable aux familles "transmetteuses". Nous avons ainsi un large spectre de configurations mais l'échantillon réuni n'est pas représentatif de l'ensemble des jeunes G3 ${ }^{(19)}$. Il n'en demeure pas moins que tous les adultes G2 interrogés parlent la langue d'origine et que chez les G3 le maintien d'un bilinguisme fonctionnel n'est pas seulement anecdotique, même en présence de couples parentaux mixtes du point de vue linguistique. Ainsi les indications d'un bilinguisme fonctionnel diffus chez les G3 confortent notre hypothèse initiale que la dynamique suisse/européenne s'écarte quelque peu de l'évolution observée outre-Atlantique.

Ces résultats confirment, certes, l'érosion de la langue d'origine/héritée maintes fois observée chez les petits-enfants de migrants, mais, en même temps, ils y apportent deux bémols. Le rythme de cette érosion parait freiné par la volonté des parents de favoriser une forme plus ou moins achevée de compétence bilingue et par la présence et la mobilisation des grands-parents à cette fin. De plus, le maintien profite d'une offre extra-familiale de formation linguistique qui consolide les connaissances acquises informellement ainsi que de la fréquence des échanges transnationaux qui alimentent et actualisent la motivation personnelle des jeunes.

Plusieurs facteurs expliquent les traits quelque peu nouveaux de la question. Lallongement de la durée de vie rend plus fréquente que par le passé la coexistence de plusieurs générations de la même famille : les grands-parents des familles italiennes 
et espagnoles sont bien disposés à assurer une présence et une prise en charge de leurs petits-enfants. La valorisation du bi/plurilinguisme dans une Europe qui s'affiche composite au plan linguistique diminue efficacement les inhibitions de parents et grands-parents quant à la transmission de la langue d'origine.

Cette volonté de transmission linguistique familiale ne saurait cependant se passer d'un support externe à la famille, à savoir les cours de langue et culture d'origine ${ }^{(20)}$ et/ou la possibilité de s'appuyer sur une offre de cours au niveau gymnasial qui permettent à nombre de jeunes d'explorer leurs choix identitaires. Dans la très grande majorité des cas l'aboutissement n'est pas une remise en question de leur inscription durable dans ce pays mais plutôt une conception de cette inscription comme étant non pas exclusive mais ouverte sur le monde.

En Europe les langues d'origine des migrants semblent assumer un rôle de marqueur identitaire plus affirmé que cela n'est le cas aux États-Unis ${ }^{(21)}$,justement à cause de la connectivité élevée entre des espaces linguistiques différents. Mais le maintien des pratiques langagières en LOH reste largement influencé par le statut des langues en présence, leur acceptation et l'accessibilité qui en découle. Dans le contexte helvétique, le statut de langue internationale de l'espagnol et de langue nationale de l'italien suggère que les bilinguismes impliquant ces langues apparaissent particulièrement légitimes et, de ce fait, volontiers pratiqués chez les petits enfants de migrants.

\section{Notes}

1. Fishman Joshua Aaron, "Language Maintenance and Language Shift as a Field of Inquiry. A Definition of the Field and Suggestions for its Further Development", in Linguistics, n 9, 1964, pp. 32-70 ; Fishman Joshua Aaron (ed.), Handbook of Language and Ethnic Identity, Oxford, Oxford University Press, 1999.

2. Hansen Marcus Lee, The Problem of the Third Generation Immigrant, Rock Island, Augustana Historical Society, 1938.

3. Nous avons bénéficié du soutien du Fonds national suisse de la recherche scientifique dans le cadre du PNR 56

Diversité des langues et compétences linguistiques en Suisse.

4. Lüdi Georges et Bernard Py, Etre bilingue. Bern, Lang, 1986.

5. Kagan Olga, "The Heritage Language Learner Survey: Report on the Preliminary Results. National Heritage Language

Resource Center", 2007.http://www.international.ucla.edu/languages/nhlrc/surveyreport

6. Héran François, "Une approche quantitative de l'intégration linguistique en France", in Hommes et Migrations, $\mathrm{n}^{\circ} 1252$, 2004,pp. 10-24.

7. Bolzman Claudio, Rosita Fibbi et Marie Vial, "Secondas - Secondos" : le processus d'intégration des jeunes issus

de la migration espagnole et italienne en Suisse, Zurich, Seismo, 2003.

8. Bolzman Claudio, Rosita Fibbi et Marie Vial, "La famille : une source de légitimité pour les immigrés après

la retraite ?", in Revue européenne des migrations internationales, vol. 17, n¹, 2001, pp. 55-78.

9. Kagitcibasi, Cigdem, "The autonomous-relational self: A new synthesis", in European Psychologist, vol. 1, n³, 1996, pp.

180-186.

10. Cela ne les empêche pas de se réjouir si, après tout, leurs enfants parviennent à comprendre le dialecte d'origine, qui reste apprécié affectivement.

11. Il y a deux sortes de couples mixtes du point de vue linguistique. Il y a des couples qui réunissent deux parents d'origine immigrée : dans ce cas, la place des deux langues d'origine dans la sphère familiale n'est guère contestée même si elles n'ont pas nécessairement la même importance. Il y a aussi des couples qui réunissent un parent d'origine immigrée et un autochtone : nous avons rencontré aussi bien le cas du parent autochtone qui soutient et valorise la pratique de la langue d'origine du parent migrant que le cas du parent autochtone qui interdit la pratique de cette langue d'origine. 12. Dans certaines situations ce sont mêmes les beaux-enfants germanophones ou francophones qui invitent les grandsparents à assumer ce rôle. 
13. Weber Chantal, "Come il nonno, così il nipote? La lingua degli immigrati di prima e di terza generazione a confronto", in Linguistica italiana, Basilea, Università di Basilea, Lavoro di licenza, 2008.

14. Alba Richard et al., "Only English by the Third Generation? Mother-Tongue Loss and Preservation among the Grandchildren of Contemporary Immigrants", Demography, n³9, 2002, pp. 467-484 ; Ishizawa Hiromi, "Minority Language Use Among Grandchildren in Multigenerational Households", in Sociological Perspectives, vol. 47, n4, 2004, pp. 465-483.

15. Wyssmüller Chantal et Rosita Fibbi, "Sprachgebrauch und Identifikationen bei italienisch- und spanischstämmigen Jugendlichen der dritten Generation in der Schweiz", in InterDIALOGOS, 2009.

16. Carli Augusto et al., "Asserting Ethnic Identity and Power Through Language", Journal of Ethnic and Migration Studies, vol. 29, n5, 2003, pp. 865-883.

17. Fibbi Rosita et Chantal Wyssmüller, "Trasmissioni familiari, pratiche linguistiche e identità dei giovani della terza generazione", Zurigo in italiano, Zurigo, 25 ottobre 2008.

18. Vatz Laaroussi Michèle, "Des ruptures migratoires à la transmission familiale, un espace de production sociale", in Vatz Laaroussi Michèle, Claudio Bolzman et Mohamed Lahlou (eds.) Familles migrantes au gré des ruptures. Tisser la transmission, Lyon, Linterdisciplinaire, 2008.

19. En particulier la fréquence à un moment ou à un autre des cours de langue et culture d'origine paraît largement supérieure à celle observée aujourd'hui auprès des jeunes G3.

20. Ces cours financés par les pays d'origine des migrants représentent une offre de formation qui s'ajoute aux structures ordinaires de la scolarité obligatoire dans le pays de résidence.

21. Waters Mary C., Ethnic Options. Choosing Identities in America, Berkeley, University of California Press, 1990. 